\title{
A SURVEY ON THE BÖTTCHER-WENZEL CONJECTURE AND RELATED PROBLEMS
}

\author{
Che-man Cheng, XiaO-QIng Jin and SeaK-Weng Vong
}

Abstract. A fundamental fact in matrix theory is that the matrix multiplication is not commutative, i.e., there are square matrices $X$ and $Y$ such that $X Y \neq Y X$. The difference $X Y-Y X$ is called the commutator (or Lie product) of $X$ and $Y$. The commutator plays an important role in diverse areas in mathematics, for instance, Lie group and Lie algebra theory, perturbation analysis, and matrix manifold computation. Böttcher and Wenzel proposed the following conjecture in 2005 : for any real $n \times n$ matrices $X$ and $Y$,

$$
\|X Y-Y X\|_{F} \leqslant \sqrt{2}\|X\|_{F}\|Y\|_{F},
$$

where $\|\cdot\|_{F}$ is the Frobenius norm. This survey is concerned with the proofs of this conjecture and the study of its related problems.

Mathematics subject classification (2010): 15A52, 15A45, 60H25, 65F35.

Keywords and phrases: Commutator of two matrices, Böttcher-Wenzel conjecture, Frobenius norm.

\section{REFERENCES}

[1] P. Absil, R. Mahony, And R. Sepulchre, Optimization Algorithms on Matrix Manifolds, Princeton University Press, Princeton, 2008.

[2] K. AUDENAERT, Variance bounds, with an application to norm bounds for commutators, Linear Algebra Appl., vol. 432 (2010), pp. 1126-1143.

[3] R. Bhatia, Matrix Analysis, Springer, New York, 1997.

[4] R. Bhatia And F. Kittaneh, Commutators, pinchings and spectral variation, Oper. Matrices, vol. 2 (2008), pp. 143-151.

[5] A. BÖTTCHER AND D. WENZEL, How big can the commutator of two matrices be and how big is it typically? Linear Algebra Appl., vol. 403 (2005), pp. 216-228.

[6] A. BötTCher AND D. WenZel, The Frobenius norm and the commutator, Linear Algebra Appl., vol. 429 (2008), pp. 1864-1885.

[7] C. Cheng, K. Fong, AND W. LEI, On some norm inequalities involving the commutator and XY$Y X^{T}$, Linear Algebra Appl., vol. 438 (2013), pp. 2793-2807.

[8] C. Cheng, K. Fong, AND I. LoK, Another proof for commutators with maximal Frobenius norm, Recent Advances in Scientific Computing and Matrix Analysis, pp. 9-14, Editors: X. Jin, H. Sun, and S. Vong, International Press, 2011.

[9] C. Cheng, S. Vong, And D. Wenzel, Commutators with maximal Frobenius norm, Linear Algebra Appl., vol. 432 (2010), pp. 292-306.

[10] K. Fong, C. Cheng, AND I. LoK, Another unitarily invariant norm attaining the minimum norm bound for commutators, Linear Algebra Appl., vol. 433 (2010), pp. 1793-1797.

[11] K. Fong, I. LOK, AND C. CHENG, A note on the norm of commutator and the norm of $X Y-Y X^{T}$, Linear Algebra Appl., vol. 435 (2011), pp. 1193-1201.

[12] R. Horn And C. Johnson, Matrix Analysis, Cambridge University Press, Cambridge, 1985.

[13] F. Kittaneh, Commutator inequalities associated with the polar decomposition, Proc. Amer. Math. Soc., vol. 130 (2002), pp. 1279-1283. 
[14] F. KitTANEH, Inequalities for commutators of positive operators, J. Funct. Anal., vol. 250 (2007), pp. $132-143$.

[15] L. LÁsZLó, Proof of Böttcher and Wenzel's conjecture on commutator norms for 3-by-3 matrices, Linear Algebra Appl., vol. 422 (2007), pp. 659-663.

[16] C. Li AND J. CUI, Map preserving product $X Y-Y X^{*}$ on factor von Neumann algebra, Linear Algebra Appl., vol. 431 (2009), pp. 833-842.

[17] Z. LU, Normal scalar curvature conjecture and its applications, J. Funct. Anal., vol. 261 (2011), pp. 1284-1308.

[18] Z. LU, Proof of the normal scalar curvature conjecture, Available from: arxiv:0711.3510v1 [math.DG] 22 November, 2007.

[19] Z. LU, Remarks on the Böttcher-Wenzel inequality, Linear Algebra Appl., vol. 436 (2012), pp. 25312535.

[20] F. TERÁN AND F. DOPICO, The solution of the equation $X A+A X^{T}=0$ and its application to the theory of orbits, Linear Algebra Appl., vol. 434 (2011), pp. 44-67.

[21] S. Vong And X. Jin, Proof of Böttcher and Wenzel's conjecture, Oper. Matrices, vol. 2 (2008), pp. $435-442$.

[22] D. Wenzel, Dominating the commutator, Oper. Theory Adv. Appl., vol. 202 (2010), pp. 579-600.

[23] D. Wenzel AND K. AUdenAERT, Impression of convexity: an illustration for commutator bounds, Linear Algebra Appl., vol. 433 (2010), pp. 1726-1759.

[24] Y. WU AND X. LIU, A short note on the Frobenius norm of the commutator, Mathematical Notes, vol. 87 (2010), pp. 935-940.

[25] X. Zhan, Matrix Theory, American Mathematical Society, Rhode Island, 2013. 